Pacific Journal of Mathematic 


\title{
A NOTE ON REAL ORTHOGONAL MEASURES
}

\author{
PATRICK AHERN AND N. V. RAO
}

\begin{abstract}
Let $X$ be an open Riemann surface and $K$ a compact subset of $X$ such that $X-K$ has only finitely many connected components. Let $R(K)$ denote the space of meromorphic functions with poles off $K$. In this note, we investigate the space of real measures supported on $\partial K$ and orthogonal to $R(K)$ and connect it with the first homology group of the interior of $K$.
\end{abstract}

1. Introduction and preliminary notations. Let $X$ be a fixed open connected Riemann surface; $K$ a compact subset of $X$ such that $X-K$ has only finitely many connected components. Let $\mathscr{C}(\partial K)$ denote the space of all real valued continuous functions on $\partial K ; \mathscr{R}(K)$ denote the space of all meromorphic functions on $X$ with poles outside $K ; \overline{\operatorname{Re} \mathscr{R}(K)}$ denote the closure of the space of real parts of functions in $\mathscr{R}(K)$ under the sup norm on $\partial K$. Let $\mathscr{C}(K)$ denote the space of all measures on $\partial K$ that are orthogonal to $\mathscr{R}(K)$ and $m(K)$ denote those measures of $\mathscr{C}(K)$ that are real.

The sole purpose of this note is to establish the following theorems.

THEOREM 1.1. There exists a natural isomorphism between $m(K)$ and the first cohomology group of $\dot{K}$ (which we shall denote by $\Omega$ hereafter) with real coefficients.

THEOREM 1.2. One can select a set of functions depending only on a homology basis of $\Omega$ in a natural way so that they form a basis for $\mathscr{C}(\partial K)$ modulo $\overline{\operatorname{Re} \mathscr{R}(K)}$.

When $X$ is the complex plane, Theorem 1.1 has already been established by Ahern and Sarason in [2] and Glicksberg in [5]. Walsh [9] already proved in this case that $\log \left|z-a_{i}\right|, 1 \leqq i \leqq n$ generate $\mathscr{C}(\partial K)$ modulo $\overline{\operatorname{Re} \mathscr{R}(K)}$ where $a_{i}$ are selected one each from the connected components of $X-K$. He also saw that they need not form a basis as in the case of the crescent moon.

The precise determination of which logarithmic terms are necessary was first given in [2] and later by Glicksberg in [5] by another method. In the case of the plane, we prove these theorems in a separate note without recourse to the techniques of uniform algebras.

2. Topological preliminaries. We need some results that are 
purely topological and we give proofs where we can not give a good reference.

THEOREM 2.1. Let $U$ be an open subset of an open Riemann surface $Y$ such that $Y-U$ has only finitely many connected components each of which is noncompact. Then the canonical homomorphism $i: H_{1}(U) \rightarrow H_{1}(Y)$ is injective where $H_{1}$ is the first homology group functor.

Proof. Let $K$ be a triangulation of $Y$ and $K^{(n)}$ denote the $n$th barycentric subdivision of $K$ and let $L^{(n)}$ denote the subcomplex made up of all those 2-simplices of $K^{(n)}$ that are contained in $U$.

Let $i_{n}: H_{1}\left(L^{(n)}\right) \rightarrow H_{1}(Y)$ be the natural homomorphism. It is enough to prove that $i_{n}$ is injective for all $n$ since $H_{1}(U)$ is the direct limit of $H_{1}\left(L^{(n)}\right)$. Writing the homology exact sequence

$$
H_{2}\left(Y, L^{(n)}\right) \longrightarrow H_{1}\left(L^{(n)}\right) \longrightarrow H_{1}(Y)
$$

we see that it is enough to prove that $H_{2}\left(Y, L^{(n)}\right)=0$. Since the considerations are the same for all $n$, we shall drop the superscript $n$. Let $z=\sum_{i=1}^{k} n_{i} s_{i}$ be any two cycle made up of simplices not in $L$ such that $z \in L$. Let $|z|$ denote the set of all points that belong to at least one of the $s_{i}$ i.e., the so-called support of $z$. We claim that the topological boundary of $|z|$ is contained in $|L|=$ support of $L$. Let $P$ be a boundary point of $|z|$ and $P \notin|L|$. But $P \in\left|\partial s_{i}\right|$ for some $i$. Let $a$ be the 1-simplex of $s_{i}$ to which $P$ belongs. By hypothesis, $a \notin L$ and since $\partial z \subset L$, this $a$ must get cancelled by another 1-simplex of $s_{j}$ for some $j \neq i$. Thus if $P$ is not a vertex of $s_{i}, P \in$ interior of $|z|$. And if $P$ is a vertex of $s_{i}$, then star of $P$ must be part of $|z|$. In either case if $P \notin|L|, P \in$ interior of $|z|$.

Also the interior of $|z|$ must contain points of $Y-U$ for otherwise $|z|$ would be contained in $U$ and hence $z \subset L$. Hence the interior of $|z|$ must intersect some connected component $C$ of $Y-U$. Since $C \cap|L|=\phi$ and boundary of $|z| \subset|L|, C \subset \mid \stackrel{z}{z}$. But then $C$ is noncompact whereas $|z|$ is compact. A contradiction!

Hence $z=0$ ie $H_{2}(Y, L)=0$.

LEMMA 2.2. $\quad H_{1}(\Omega)$ is finitely generated.

Proof. We can suitably shrink the ambient Riemann surface $X$ to $X_{0}$ so that $K \subset X_{0}, X_{0}-K$ has finitely many connected components each of which is noncompact and further $H_{1}\left(X_{0}\right)$ is a free Abelian group of finite rank.

By the preceding theorem, $H_{1}(\Omega)$ is a subgroup of $H_{1}\left(X_{0}\right)$ and 
hence is a free Abelian group of finite rank.

For complete details regarding barycentric subdivisions, homology groups etc. one can confer [3], Ch. I.

LEMMA 2.3. Let $Y$ be a connected open Riemann surface and assume $H_{1}(Y)$ is finitely generated. Then there exists a subregion $\Omega_{0}$ relatively compact and bounded by simple closed curves $\gamma_{1}, \gamma_{2}, \cdots$, $\gamma_{k}$ such that every component of $Y-\bar{\Omega}_{0}$ is an annulus.

Proof. Canonical form of $Y$ (see [3], p. 94) is (let us say) with $p$ handles and $q$ contours i.e., by cutting out $2 p+q$ discs out of the Riemann sphere and then attaching $p$ handles by pairing off $2 p$ of the holes, we get a homeomorph of $Y$.

Thus by taking off $q$ ringed domains one around each hole, we get a subregion $\Omega_{0}$ such that every connected component of $Y-\bar{\Omega}_{0}$ is an annulus.

DEFINITION 2.4. Let $U$ be an open subset of a Riemann surface $X$. A path at $x$ in $U$ is a Jordan arc entirely lying in $U$ except possibly at one endpoint which is $x$ when $x \in \partial U$.

Two paths at $x$ in $U$ are said to be equivalent if and only if given any neighborhood $N$ of $x$, there exists an arc joining the two paths and lying entirely in $N \cap U$. A point $x$ is said to be a multiple point of $U$ if there exist two inequivalent paths at $x$ in $U$.

LEMMA 2.5. Let $K$ be a compact subset of an open connected Riemann surface $X$ such that $X-K$ has only finitely many connected components. Let $\Omega=\stackrel{\circ}{K}$. The set of multiple points of $\Omega$ is countable and given any multiple point $x$ of $\Omega$, there exists at most countably many inequivalent paths at $x$ in $\Omega$.

Proof. Let $x_{0} \in \partial \Omega$. Since $X-K$ has only finitely many connected components, there exists a closed parametric disc $\Delta$ with center at $x_{0}$ such that no connected component of $X-K$ is completely contained in $\Delta$.

Let $\phi: \Delta \rightarrow C$ denote the coordinate mapping and $C$, the image of $\Delta \cap K$ by $\phi . \quad C$ is compact and the complement of $C$ is connected since any connected component of $X-K$ that intersects $\Delta$ would have points on the $\operatorname{rim}$ of $\Delta$. Thus any multiple point of $\Omega$ contained in the interior of $\Delta$ is mapped into a multiple point of $\dot{C}$ and further any two inequivalent paths at $x$ in $\Omega$ are mapped to inequivalent paths at $\phi(x)$ in $\stackrel{\circ}{C}$.

Just for this discussion alone, let us make the convention that 
capital letters denote paths and small letters their extremeties. Thus $x P y$ shall denote a path $P$ with extremeties $x, y$ and oriented from $x$ to $y$.

Now let $x P_{1} y_{1}, x P_{2} y_{2}$ be two paths at $x$ in $\dot{C}$ and $x$ a multiple point of $\dot{C}$. Assume further that these two paths lie in the same connected component $U$ of $\dot{C}$. We join these two paths by a path $y_{1} Q y_{2}$ completely contained in $U$. Then $x P_{1} y_{1} Q y_{2} P_{2} x$ is a Jordan curve completely contained in $U$ but for the point $x$. Certainly the interior of this curve must be completely contained in $\stackrel{\circ}{C}$ for otherwise it would intersect the complement of $C$ thus trapping a connected component of the complement of $C$. But complement of $C$ is connected and unbounded leading to a contradiction. Thus $x P_{1} y_{1} Q y_{2} P_{2} x$ is the boundary of a Jordan domain contained in $U$. But Jordan domains are locally arc-wise connected even at the boundary (see Goluzin [6], p. 46). Hence $x P_{1} y_{1}$ and $x P_{2} y_{2}$ are equivalent paths at $x$ in $\stackrel{\circ}{\text {. }}$.

This proves that two paths are inequivalent if and only if they are contained in different connected components of $\stackrel{\circ}{C}$. Thus the number of inequivalent paths at a point $x$ does not exceed the number of connected components of $\dot{C}$ and hence they are at most countable.

Now let $U_{1}, U_{2}$ be two connected components of $\dot{C}$ and let $x, u$ belong to $\partial U_{1} \cap \partial U_{2}, x P_{1} y_{1}, x P_{2} y_{2}$ be paths at $x$ in $U_{1}$ and $U_{2}$ respectively and $u Q_{1} z_{1}, u Q_{2} z_{2}$ be paths at $u$ in $U_{1}$ and $U_{2}$ respectively. Let $y_{1} R_{1} z_{1}, y_{2} R_{2} z_{2}$ be two paths lying entirely in $U_{1}$ and $U_{2}$ respectively. Now interior of the Jordan curve $x P_{1} y_{1} R_{1} z_{1} Q_{1} u Q_{2} z_{2} R_{2} y_{2} P_{2} x$ must trap a component of the complement of $\dot{C}$ for otherwise it would be completely contained in $C$ and hence in $\dot{C}^{\circ}$ joining $U_{1}$ and $U_{2}$ which is impossible. This means that given any multiple point $x$ of $\dot{C}$, we can associate a pair of coonected components of $\dot{C}$ where the inequivalent paths to $x$ in $C$ come from and this association is one-to-one. Since the number of connected components of $\dot{C}$ is at most countable, we obtain that the set of multiple points of $\dot{C}$ is also at most countable.

Since $K$ can be covered by the interiors of a finite number of parametric discs, the lemma is proved.

LEMmA 2.6. Let $\Delta$ denote the annulus $\delta<|z|<1$ and $\phi: \Delta \rightarrow U$ be a conformal isomorphism and $U$ be a relatively compact subset of a connected open Riemann surface $X$. Assume $\partial U=C \cup D$ where $C$ and $D$ are both compact and disjoint.

Let $\phi(|z|=\delta)$ denote the set of all points $\zeta$ in $X$ for which 
there exists a sequence $z_{n} \in \Delta,\left|z_{n}\right| \rightarrow \delta$ as $n \rightarrow \infty$ and $\dot{\phi}\left(z_{n}\right) \rightarrow \zeta$ as $n \rightarrow \infty$. By analogy, we can define $\dot{\phi}(|z|=1)$.

Then $\dot{\phi}(|z|=1), \dot{\phi}(|z|=\delta)$ are both connected and either $\dot{\phi}(|z|=1)=$ $C, \dot{\phi}(|z|=\delta)=D$ or $\dot{\phi}(|z|=\delta)=C, \phi(|z|=1)=D$.

Proof. Evidently $\phi(|z|=\delta)$ is a closed set in $X$. Assume that $\dot{\phi}(|z|=\delta)$ is disconnected i.e., $\phi(|z|=\delta)=A_{1} \cup A_{2}$ where $A_{1}, A_{2}$ are mutually disjoint nonempty closed sets in $X$. Then there exist open sets $V_{1}, V_{2}$ such that $V_{i} \supset A_{i}, i=1,2$ and $V_{1} \cap V_{2}=\phi$. We claim that $\phi(\delta<|z|<r) \subset V_{1} \cup V_{2}$ for all $r$ sufficiently close to $\delta$. If not, there exists a sequence $r_{n} \downarrow \delta$ and $z_{n}$ with $\left|\boldsymbol{z}_{n}\right|=r_{n}$ and $\phi\left(\boldsymbol{z}_{n}\right) \notin V_{1} \cup V_{2}$.

This is impossible since on the one hand all limit points of $\dot{\phi}\left(z_{n}\right)$ would belong to $\phi(|z|=\delta)$ and on the other hand should lie outside $V_{1} \cup V_{2}$ which is an open set containing $\phi(|z|=\delta)$.

Since $\phi(\delta<|z|<r)$ is connected, the fact that $\phi(\delta<|z|<r) \subset$ $V_{1} \cup V_{2}$ implies that $\dot{\phi}(\delta<|z|<r) \subset V_{1}$ or $V_{2}$ which means that $\phi(|z|=\delta) \subset \bar{V}_{1}$ or $\bar{V}_{2}$. Since $\bar{V}_{1} \cap V_{2}=\bar{V}_{2} \cap V_{1}=\phi, \phi(|z|=\delta) \cap V_{2}=$ $\dot{\phi}$ or $\phi(|z|=\delta) \cap V_{1}=\phi$. That is impossible. Hence $\phi(|z|=\delta)$ is connected. Similarly $\phi(|z|=1)$ is connected.

Further any boundary point of $U$ must belong either to $\dot{\phi}(|z|=\delta)$ or $\phi(|z|=1)$. Let $\xi_{0} \in \partial U$ and $\left\{\zeta_{n}\right\}$ be a sequence of points in $U$ such that $\zeta_{n} \rightarrow \zeta_{0}$ as $n \rightarrow \infty$. Then if $\phi\left(z_{n}\right)=\zeta_{n}, z_{n} \in \Delta$, any limit point $z_{0}$ of $\left\{z_{n}\right\}$ must belong to $\partial \Delta$. For if not, $z_{n_{k}} \rightarrow z_{0}$ as $k \rightarrow \infty$ and $z_{0} \in \Delta$ and $\phi\left(z_{n_{k}}\right)=\zeta_{n_{k}} \rightarrow \zeta_{0}=\phi\left(z_{0}\right)$ as $k \rightarrow \infty$. But $\phi\left(z_{0}\right)$ is an interior point of $U$ and $\zeta_{0}$ is a boundary point of $U$. A contradiction. A similar reasoning would prove that $\phi(\partial \Delta) \subset \partial U$. Consequently $\phi(\partial \Delta)=\partial U$.

This proves that $\partial U$ has at most two connected components. By hypothesis $\partial U$ has at least two connected components. Hence $C$ and $D$ must be connected and $\phi(|z|=1), \phi(|z|=\delta)$ must be disjoint.

Hence $\phi(|z|=1)=C$ and $(|z|=\delta)=D$ or $\phi(|z|=1)=D$ and $\phi(|z|=\delta)=C$.

LEMMA 2.7. Hypothesis and notation same as in the previous lemma. There exists a Borel set $E \subset[0,2 \pi]$ of length $2 \pi$ such that $\lim _{r \rightarrow 1} \phi\left(r e^{i \theta}\right), \lim _{r \rightarrow i} \phi\left(r e^{i \theta}\right)$ exist for all $\theta \in E$.

Proof. Narasimhan [8] proved that any open Riemann surface can be imbedded in $C^{3}$ as a closed sub-manifold. Hence there exist three holomorphic functions $\psi_{i}, i=1,2,3$ such that $\psi(\zeta)=\left(\psi_{1}(\zeta)\right.$, $\left.\psi_{2}(\zeta), \psi_{3}(\zeta)\right)$ from $X \rightarrow C^{3}$ is a one-one holomorphic map.

Since $\bar{U}$ is compact, $\psi / U$ is bounded and hence $\psi_{i}^{\circ} \circ$ is bounded for $i=1,2,3$. By Fatou's theorem (see [10] pp. 99-100) on radial 
limits, there exists a Borel set $E \subset[0,2 \pi]$ of length $2 \pi$ such that $\lim _{r \rightarrow 1} \psi_{i} \circ \dot{\phi}\left(r e^{i \theta}\right), \lim _{r \rightarrow \dot{\theta}} \psi_{i} \circ \dot{\phi}\left(r e^{i \theta}\right)$ exist for all $\theta \in E, i=1,2,3$.

Let $\theta \in E, r_{n} \uparrow 1$ and $\phi\left(r_{n} e^{i \theta}\right) \rightarrow \zeta_{0}$ as $n \rightarrow \infty$. Then $\lim _{n \rightarrow \infty} \eta^{\prime} r_{i}$. $\dot{\phi}\left(r_{n} e^{i \theta}\right)=\dot{\psi}_{i}\left(\zeta_{0}\right)=\lim _{r \rightarrow 1} \dot{\psi} \dot{\psi}_{2} \circ \phi\left(r e^{i \theta}\right)$ for $i=1,2,3$. Since $\psi$ is $1-1$, this shows that $\zeta_{0}$ does not depend on the sequence $\left\{r_{n}\right\}$. Hence $\lim _{r \rightarrow 1} \phi\left(r e^{i \theta}\right)$ exists. Similarly $\lim _{r \rightarrow o} \phi\left(r e^{i \theta}\right)$ exists for all $\theta \in E$.

Lemma 2.8. Hypothesis same as in Lemma 2.6. Further assume that $X-\bar{U}$ has only finitely many connected components. Then by discarding a countable subset of $E$ ( $E$ as in Lemma 2.7), we can assume that $\theta \rightarrow \lim _{r \rightarrow 1} \dot{\phi}\left(r e^{20}\right)$ and $\theta \rightarrow \lim _{r \rightarrow \dot{o}} \phi\left(r e^{i \theta}\right)$ are both one-one on $E$.

Proof. Let $\theta \in E, P_{0}$ denote the path $\dot{\phi}\left(r e^{i \theta}\right), 1-\varepsilon<r<1$, $\varepsilon$ a fixed small positive number; $\zeta_{\theta}=\lim _{r \rightarrow 1} \phi\left(r e^{i \theta}\right)$.

Now if $\theta_{1} \neq \theta_{2}$ and $\zeta_{\theta_{1}}=\zeta_{O_{2}}$, then $\zeta_{\theta_{1}}$ is a multiple point and $P_{\theta_{1}}, P_{\theta_{2}}$ are inequivalent (see [6], pp. 38-39). Thus $\zeta_{\theta_{1}}$ is a multiple point of $U$. By Lemma 2.5, the set of multiple points is countable and at any given multiple point, there can be at most countably many inequivalent paths.

Thus given a $\theta_{0} \in E$, the set of all $\theta \in E, \theta \neq \theta_{0}, \zeta_{0}=\zeta_{\theta_{0}}$ is countable; further the set of all $\theta_{0}$ for which there exists a $\theta \neq \theta_{0}$ such that $\zeta_{0}=\zeta_{\theta_{0}}$ is also countable. Hence by discarding all such $\theta_{0}$ out of $E$, we obtain a new Borel set $E$ of length $2 \pi$ such that $\theta \rightarrow \lim _{r \rightarrow 1} \phi\left(r e^{i \theta}\right)$ is a $1-1$ map. A similar reasoning applied as $r \rightarrow \delta$ would prove the rest of the lemma.

\section{Boundary measures and analytic differentials.}

Definition 3.1. Let $U$ be an open subset of a connected open Riemann surface $X$. An increasing sequence $\left\{U_{n}\right\}$ of open sets is said to be a regular exhaustion of $U$ if $U_{n}$ is a relatively compact subset of $U_{n+1}$ for all $n$; $\bigcup_{n=1}^{\infty} U_{n}=U ; \partial U_{n}$ consists of finitely many piecewise analytic Jordan curves and $U-\bar{U}_{n}$ has no relatively compact connected components in $U$.

REMARK. Existence of regular exhaustions can be proved by triangulations (see [3], pp. 62-63).

Definition 3.2. Let $U$ be an open subset of $X$. $\mathscr{C}(U)$ denotes the set of all holomorphic 1-forms $\omega$ for which there exists a regular exhaustion $\left\{U_{n}\right\}$ of $U$ such that $\int_{\partial^{U}{ }_{n}}|\omega| \leqq c$ where $c$ is independent of $n$. 
DEFINITION 3.3. Let $U$ be a relatively compact open subset of an open connected Riemann surface $X$. Let $\omega \in \mathscr{H}(U)$. A finite Borel measure $\mu$ on $\partial U$ is called a boundary measure of $\omega$ if there exists a regular exhaustion $U_{n}$ of $U$ such that $\int_{\partial U_{n}} h \omega \rightarrow \int_{\partial U} h d_{\mu}$ as $n \rightarrow \infty$ for any continuous function $h$ on $\bar{U}$ where $\partial U_{n}$ is positively oriented with respect to $U_{n}$.

THEOREM 3.4. (Bishop-Kadama, see [7]). Let $K$ be a compact subset of $X$ such that $X-K$ has only finitely many connected components. Let $\stackrel{\circ}{K}=\Omega$. Given any $\omega \in \mathscr{K}(\Omega)$, there exists one and only one boundary measure $\mu_{\omega}$ of $\omega$.

The mapping $\omega \rightarrow \mu_{\omega}$ is a linear isomorphism between $\mathscr{H}(\Omega)$ and $\mathscr{C}(K)$ (see $\S 1$ for the definition of $\mathscr{C}(K)$ ).

Definition 3.5. Let $U$ be an open subset of $X$. A point $x \in \partial U$ is said to be an accessible boundary point of $U$ if and only if there exists a path at $x$ in $U$. Acc $\partial U$ shall denote the set of all accessible boundary point of $U$.

THEOREM 3.6. Let $K$ be a compact subset of $X$ and $X-K$ have only finitely many connected components. Let $\stackrel{\circ}{K}=\Omega$. Let $\left\{U_{i}, i \in I\right\}$ be the family of all connected components of $\Omega$. By Lemma 2.2, $H_{1}(\Omega)$ is finitely generated and consequently $H_{1}\left(U_{i}\right)$ is finitely generated for all $i \in I$. By Lemma 2.3, there exists a relatively compact subregion $V_{i}$ of $U_{i}$ bounded by finitely many analytic Jordan curves such that each component of $U_{i}-\bar{V}_{i}$ is an annulus. Let $\left\{\Delta_{i j}, 1 \leqq j \leqq N(i)\right\}$ denote the set of all connected components of $U_{i}-\bar{V}_{i}$. Let $\omega \in \mathscr{H}(\Omega)$.

Then $\omega / \Delta_{i j} \in \mathscr{P}\left(\Delta_{i j}\right)$. Let $\mu_{i j}$ denote the boundary measure of $\omega / \Delta_{i j}$ located on $\partial \Delta_{i j} \cap \partial \Omega$. Then $\mu_{i j}, \mu_{i^{\prime} j^{\prime}}$ are mutually singular for $(i, j) \neq\left(i^{\prime}, j^{\prime}\right)$. Further $\sum_{i \in I} \sum_{1 \leqq j \leqq N(i)}\left\|\mu_{i j}\right\|$ is finite and $\mu_{\omega}=$ $\sum_{i} \sum_{j} \mu_{i j}$.

Before proceeding to the proof of the Theorem 3.6, we need two lemmas.

Lemma 3.7. Let $\Delta$ denote the annulus $\{z ; \delta<|z|<1\}$ and $\omega \in$ $\mathscr{H}(\Delta)$. Let $\omega=f(z) d z$ where $f$ is holomorphic in $\Delta$. Then there exists a Borel measurable function $f$ defined on $\partial \Delta$ such that

$$
\lim _{r \rightarrow 1-0} \int_{0}^{2 \pi}\left|f\left(r e^{i \theta}\right)-f\left(e^{i \theta}\right)\right| d \theta=0 \text { and } \lim _{r \rightarrow \dot{0}+0} \int_{0}^{2 \pi} \mid f\left(r e^{i \theta}\right)-f\left(\delta e^{i \theta}\right) d \theta=0 .
$$

Proof. Let $\mu$ denote the boundary measure $\mu_{\omega}$ of $\omega$. 


$$
\text { Let } f_{1}(z)=\int_{|\zeta|=1} \frac{d \mu(\zeta)}{\zeta-z} \text { and } f_{2}(z)=\int_{|\zeta|=\delta} \frac{d \mu(\zeta)}{\zeta-z}
$$

so that $f_{1}$ is holomorphic in $|z|<1$ and $f_{2}$ is holomorphic in $|z|>\delta$ and $f=f_{1}+f_{2}$ in $\Delta$.

Let $\nu_{1}, \nu_{2}$ be finite complex Borel measures defined by

$$
\begin{aligned}
& d \nu_{1}(\zeta)=d \mu(\zeta)-\frac{1}{2 \pi i} f_{2}(\zeta) d \zeta \text { on }|\zeta|=1 \\
& d \nu_{2}(\zeta)=d \mu(\zeta)+\frac{1}{2 \pi i} f_{1}(\zeta) d \zeta \text { on }|\zeta|=\delta
\end{aligned}
$$

Then for $\delta<|z|<1$,

$$
\begin{aligned}
\int \frac{d \nu_{1}(\zeta)}{\zeta-z} & =\int_{|\zeta|=1} \int \frac{d \mu(\zeta)}{\zeta-z}-\frac{1}{2 \pi i} \int_{|\zeta|=1} \frac{f_{2}(\zeta) d \zeta}{\zeta-z} \\
& =f_{1}(z)-\frac{1}{2 \pi i} \int_{\left|\zeta^{\prime}\right|=\delta} \int_{|\zeta|=1} \frac{d \mu\left(\zeta^{\prime}\right) d \zeta}{\left(\zeta^{\prime}-\zeta\right)(\zeta-z)} \\
& =f_{1}(z)
\end{aligned}
$$

since

$$
\int_{|\zeta|=1} \frac{d \zeta}{\left(\zeta^{\prime}-\zeta\right)(\zeta-z)}=0 \text { when }\left|\zeta^{\prime}\right|<1 \text { and }|z|<1 .
$$

By analytic continuation, we get that

$$
\int \frac{d \nu_{1}(\zeta)}{\zeta-z} \equiv f_{1}(z) \text { for }|z|<1
$$

Further for $|z|>1$,

$$
\int \frac{d \nu_{1}(\zeta)}{\zeta-z}=f_{1}(z)+f_{2}(z)
$$

since

$$
\int_{|\zeta|=1} \frac{d \zeta}{\left(\zeta^{\prime}-\zeta\right)(\zeta-z)}=-2 \pi i /\left(\zeta^{\prime}-z\right)
$$

Therefore

$$
\begin{aligned}
\int \frac{d \nu_{1}(\zeta)}{\zeta-z} & =f_{1}(z) \text { for }|z|<1 \\
& =0 \text { for }|z|>1
\end{aligned}
$$

By F. and M. Riesz theorem ([4], for a very general form), we 
obtain that

$$
\int_{0}^{2 \pi}\left|f_{1}\left(r e^{i \theta}\right)-f_{1}\left(r^{\prime} e^{i \theta}\right)\right| d \theta \longrightarrow 0 \text { as } r, r^{\prime} \longrightarrow 1
$$

Now by a similar reasoning, we find that

$$
\begin{aligned}
\int \frac{d \nu_{2}(\zeta)}{\zeta-z} & =f_{2}(z) \text { for }|z|>\delta \\
& =0 \text { for }|z|<\delta .
\end{aligned}
$$

Applying an inversion and $\mathrm{F}$ and $\mathrm{M}$. Riesz theorem, we obtain that

$$
\int_{0}^{2 \pi}\left|f_{2}\left(r e^{i \theta}\right)-f_{2}\left(r^{\prime} e^{i \theta}\right)\right| d \theta \longrightarrow 0 \text { as } r, r^{\prime} \longrightarrow \delta .
$$

This together with completeness of $L^{1}([0,2 \pi])$ proves our lemma.

DeFinition 3.8. Let $\phi: X \rightarrow Y$ be a holomorphic map where $X$ and $Y$ are Riemann surfaces. Then for any holomorphic 1-form $\omega$ on $Y, \phi^{*} \omega$ denotes the holomorphic 1-form defined as follows: for any $p \in X$ and a coordinate function $\zeta$ in a neighborhood $N$ of $\phi(P), \phi^{*} \omega=f(\zeta \circ \phi) d \zeta \circ \phi$ where $\omega=f(\zeta) d \zeta$ in a neighborhood of $\zeta \circ \phi(p)$.

Definition 3.9. Let $X, Y$ be two measurable spaces and $\phi: X \rightarrow$ $Y$ be a measurable map. For any measure $\mu$ on $X, \phi_{*} \mu$ denotes the measure defined by $\left(\phi_{*} \mu\right)(S)=\mu\left(\phi^{-1}(S)\right)$ for any measurable subset $S$ of $Y$.

LEMMA 3.10. Let $A_{i j}$ be as introduced in Theorem 3.6 and $\phi: \Delta \rightarrow \Delta_{i j}$ be a conformal isomorphism where $\Delta=\{z ; \delta<|z|<1\}$ and $\delta$ depends on $i, j$.

Let $B$ denote the set of all points $z$ on $\partial \Delta$ for which $\lim _{r \rightarrow 1-0} \phi(r \cdot z)$ or $\lim _{r \rightarrow \dot{o}+0} \phi(r z)$ exists and let us extend $\phi$ to $B$ by these limits. Let $\omega \in \mathscr{H}\left(\Delta_{i j}\right)$. Then $\phi^{*} \omega \in \mathscr{H}(\Delta)$ and if $\nu$ is the boundary measure of $\phi^{*} \omega$, there exists a Borel subset $B_{0}$ of $B$ on which $\nu$ is supported and $\phi_{*}(\nu)$ is the boundary measure of $\omega$.

Proof. If $\left\{U_{n}\right\}$ is a regular exhaustion of $\Delta_{i j}$, then $\left\{\phi^{-1}\left(U_{n}\right)\right\}$ is a regular exhaustion of $\Delta$ and further

$$
\int_{\partial \phi^{-1}\left(U_{n}\right)}\left|\phi^{*} \omega\right|=\int_{\partial U_{n}}|\omega| \text {. }
$$

Consequently by definition, $\phi^{*} \omega \in \mathscr{C}(\Delta)$. By Lemma 3.7, if $\phi^{*} \omega=$ 
$f(z) d z$; we can extend $f$ as a Borel measurable function to $\Delta$ such that

$$
\begin{aligned}
& \lim _{r \rightarrow 1} \int_{0}^{2 \pi}\left|f\left(r e^{i \theta}\right)-f\left(e^{i \theta}\right)\right| d \theta=0 \quad \text { and } \\
& \lim _{r \rightarrow \delta} \int_{0}^{2 \pi}\left|f\left(r e^{i \theta}\right)-f\left(\delta e^{i \theta}\right)\right| d \theta=0 .
\end{aligned}
$$

In view of Lemma 2.8 there exists a Borel set $E \subset[0,2 \pi]$ of measure $2 \pi$ such that $\lim _{r \rightarrow 1} \phi\left(r e^{i \theta}\right), \lim _{r \rightarrow \delta} \phi\left(r e^{i \theta}\right)$ exist for all $\theta \in E$. Let $B_{0}$ denote the set $\left\{z ; z=e^{i \theta}\right.$ or $\delta e^{i \theta}$ for some $\left.\theta \in E\right\}$. Obviously $B_{0}$ is a Borel set and $\phi$ can be extended by radial limits to $\Delta \cup B_{0}$ as a Borel measurable function.

The above considerations imply that if $h$ is any continuous function on $\bar{\Delta}_{i j}$.

$$
\begin{aligned}
& \lim _{r \rightarrow 1} \int_{|z|=r} h \circ \phi(z) f(z) d z=\lim _{r \rightarrow 1} \int_{\phi(|z|=r)} h \omega \text { and } \\
& \lim _{r \rightarrow \delta} \int_{|z|=r} h \circ \phi(z) f(z) d z=\lim _{r \rightarrow \delta} \int_{\phi(|z|=r)} h \omega
\end{aligned}
$$

exist and are respectively equal to

$$
\int_{B_{0} \cap|z|=1} h \circ \phi\left(e^{i \theta}\right) f\left(e^{i \theta}\right) d e^{i \theta} \text { and } \int_{B_{0} \cap|z|=\delta} h\left(\delta e^{i \theta}\right) f\left(\delta e^{i \theta}\right) d \delta e^{i \theta}
$$

for any continuous function $h$ on $\partial \Delta$.

Let us define the boundary measure $\nu$ on $\partial \Delta$ as follows: $d \nu=$ $f\left(e^{i \theta}\right) d e^{i \theta}$ on $|z|=1$ and $d \nu=-f\left(\delta e^{i \theta}\right) d \delta e^{i \theta}$ on $|z|=\delta$. Because of $(1), \nu$ is the boundary measure of $\phi^{*} \omega$ and because of (2),

$$
\int_{\partial \Delta} h \phi d \nu=\lim _{n \rightarrow \infty} \int_{\partial V_{n}} h \omega=\int_{\partial_{i} \Delta_{j}} \int h d \phi_{*} \nu
$$

where $V_{n}=\phi(\{z ; \delta+1 / n<|z|<1-1 / n\})$. Since $\left\{V_{n}\right\}$ is a regular exhaustion of $\Delta_{i j}$, by the Theorem 3.4 follows that $\phi_{*} \nu$ is indeed the boundary measure of $\omega$ on $\Delta_{i j}$.

REMARK 3.11. Boundary measure of $\omega$ is supported on acc $\partial \Delta_{i j}$ and any countable set is a null set for this measure.

Proof of Theorem 3.6. By Remark 3.11, it follows that $\mu_{i j}$ is supported on a Borel set contained in $\operatorname{acc} \partial \Delta_{i j} \subset \operatorname{acc} \partial U_{i}$ and any countable set has measure zero.

Now fixing $i$, acc $\partial \Delta_{i j} \cap \operatorname{acc} \partial \Delta_{i j^{\prime}}$ is countable for $j \neq j^{\prime}$ thanks to Lemma 2.5. Hence $\mu_{i j}, \mu_{i j^{\prime}}$ are mutually singular.

Let us assume $i \neq i^{\prime}$. The support of $\mu_{i j}$ and support of $\mu_{i^{\prime} j^{\prime}}$ are respectively contained in acc $\partial U_{i}$ and acc $\partial U_{i}^{\prime}$. By Lemma 2.5, $\operatorname{acc} \partial U_{i} \cap \operatorname{acc} \partial U_{i}$, is at most countable and by Remark 3.11 follows 
that $\mu_{i j}, \mu_{i^{\prime} j^{\prime}}$ are mutually singular.

Let $\mu_{i}$ denote the boundary measure of $\omega$ restricted to $U_{i}$. We shall now prove that $\mu_{i}=\sum_{j=1}^{N(i)} \mu_{i j}$. The boundary of $\Delta_{i j}$ falls into two parts, a Jordan curve $\gamma_{i j}$ contained in $U_{i}$ and $\partial \Omega \cap \partial \Delta_{i j}$ which of course are disjoint closed sets. Thus as in lemma 3.10, $\phi:\{z ; \delta<$ $|z|<1\} \rightarrow \Delta_{i j}$ is a conformal isomorphism, by lemma 2.6 the limit sets $\phi(|z|=\delta)$ and $\phi(|z|=1)$ are disjoint and must coincide with $\gamma_{i j}$ and $\partial \Omega \cap \partial \Delta_{i j}$ is some order. We can assume without loss of generality that $\phi(|z|=1)=\partial \Omega \cap \partial \Delta_{i j}$. Let $\gamma_{i j n}$ denote the Jordan curve $\phi(|z|=1-1 / n)$ oriented positively with respect to $\phi(\delta<|z|<$ $1-1 / n)$. For any fixed $n$ and $i,\left\{\gamma_{i j n}\right\}_{1 \leqq j \leqq N(i)}$ bound a domain $U_{i n}$ contained in $U_{i}$ and further for any continuous function $h$ on $\bar{\Omega}$,

Hence

$$
\lim _{n \rightarrow \infty} \int_{r_{i j n}} h \omega=\int h d \mu_{i j} \text { because of Lemma 3.10. }
$$

$$
\lim _{n \rightarrow \infty} \int_{\partial U_{i n}} h \omega=\sum_{j=1}^{N(i)} \int h d \mu_{i j},
$$

i.e., $\mu_{i}=\sum_{j=1}^{N(i)} \mu_{i j}$. This also proves that $\mu_{i}, \mu_{i^{\prime}}$ are mutually singular if $i \neq i^{\prime}$. Now we shall prove that $\sum_{i \in I}\left\|\mu_{i}\right\|<\infty$.

Since $\omega \in \mathscr{H}(\Omega)$, it follows that there exists a regular exhaustion $\left\{\Omega_{n}\right\}$ of $\Omega$ such that

$$
\int_{\partial \cdot 2_{n}}|\omega| \leqq C \text { where } C \text { does not depend on } n \text {. }
$$

Further for any $h$ continuous on $\bar{\Omega}, \int_{\partial \Omega_{n}} h \omega \rightarrow \int h d \mu_{\omega}$ as $n \rightarrow \infty$.

Let $F$ be a finite subset of $I$ and let $U_{F}=\bigcup_{i \in F} U_{i}$. Now from the above considerations, we obtain that $\int_{\partial\left(\Omega_{n} \cap U_{F}\right)}|\omega| \leqq C$ for all $n$ and by weak compactness of measures follows that by passing to a subsequence if necessary that $\int_{\partial\left(\Omega_{n} \cap U_{F}\right)} h \omega \rightarrow \int h d \mu_{F}$ as $n \rightarrow \infty$ where $\mu_{F}$ is the boundary measure of $\omega$ restricted to $U_{F}$. Hence $\left\|\mu_{F}\right\|<$ C. But since as $n \rightarrow \infty, \int_{\partial\left(\cup_{\left.i \in F^{U} i n\right)}\right.} h \omega=\sum_{i \in F} \sum_{j=1}^{N(i)} \int_{r_{i i n}} h \omega \rightarrow \sum_{i \in F} \int h d \mu_{i}$ and $\left\{\bigcup_{i \in F} U_{i n}\right\}$ is a regular exhaustion of $U_{F}$, we see that $\sum_{i \in F} \mu_{i}$ is also a boundary measure of $\omega / U_{F}$. By Theorem 3.4, $\sum_{i \in F} \mu_{i}=\mu_{F}$.

Consequently $\left\|\sum_{i \in F} \mu_{i}\right\| \leqq C$ for an arbitrary finite subset $F$ of $I$ and now by the fact that $\mu_{i}$ are mutually singular, we obtain that $\sum_{i \in I}\left\|\mu_{i}\right\| \leqq C$.

Now if $\mu^{\prime}=\sum_{i \in I} \mu_{i}$, we can prove that any function $f$ meromorphic on $X$ with poles off $\partial K, \int f d \mu^{\prime}=\int f d \mu_{\omega}$. It is enough to prove for a function with one pole. If the pole is not in $\Omega$, it is immediate that $\int_{\partial \Omega_{n}} f \omega=0$ and $\int_{\partial U_{i n}} f \omega=0$ for all $i$ and $n$. Hence 
$\int f d \mu^{\prime}=\int f d \mu_{\omega}=0$. Now if the pole is in some $U_{i}$, then $\int_{\partial \Omega_{n}} f \omega=$ $\int_{\partial U_{i n}} f \omega$ provided the pole is in $\Omega_{n} \cap U_{i n}$. Hence by going to the limits, $\int f d \mu_{\omega}=\int f d \mu_{i}$ and of course $\int f d \mu_{j}=0$ for $j \neq i$.

Thus $\int f d\left(\mu^{\prime}-\mu_{\omega}\right)=0$ for all functions meromorphic with poles off $\partial K$. By a theorem of Kodama (see [7]), we obtain $\mu^{\prime} \equiv \mu_{\omega}$.

Thus $\mu=\sum_{i \in I} \mu_{i}=\sum_{i \in I} \sum_{j=1}^{N(i)} \mu_{i j}$.

CoROLlaRY 3.12. Let $\bar{U}_{i}=K_{i}$. Given $\mu_{i} \in \mathscr{M}\left(K_{i}\right)$ such that $\sum\left\|\mu_{i}\right\|<\infty$, then $\sum \mu_{i} \in \mathscr{M}(K)$. Further, $\mu_{i}$ are mutually singular. Conversely given any $\mu \in \mathscr{M}(K), \mu$ can be uniquely expressed as $\sum \mu_{i}$ where $\mu_{i} \in \mathscr{C}\left(K_{i}\right)$ and $\sum\left\|\mu_{i}\right\|<\infty$.

Proof. By Theorem 3.6 $\mu_{i}$ is supported on a Borel set contained in acc $\partial U_{i}$ and any countable set is a null set modulo $\mu_{i}$. By Lemma 2.5, $\operatorname{acc} \partial U_{i} \cap \operatorname{acc} \partial U_{j}$ is a countable set and consequently, $\mu_{i}$ and $\mu_{j}$ are mutually singular.

Since $\int f d \mu_{i}=0$ for any $f$ continuous on $K_{i}$ and analytic in $U_{i}$, $\int f d \mu_{i}=0$ for any $f$ continuous on $K$ and analytic in $\Omega$. Therefore $\mu_{i} \in \mathscr{C}(K) \forall i$ and $\sum \mu_{i} \in \mathscr{M}(K)$.

For the converse, the fact that $\mu=\sum \mu_{i}, \mu_{i} \in \mathscr{C}\left(K_{i}\right)$ is a consequence of Theorem 3.6. Uniqueness follows from mutual singularity.

CoRollary 3.13. Assume that $m\left(K_{i}\right) \equiv H^{1}\left(U_{i}\right) \forall i \in I . \quad$ Then $m(K) \equiv H^{1}(\Omega)$.

Proof. $H^{1}(\Omega)$ is finitely generated by Lemma 2.2. Hence $H^{1}\left(U_{i}\right)=0$ but for finitely many $i$. The set of $i$ for which $H^{\prime}\left(U_{i}\right) \neq$ 0 , we shall denote by $F$.

Then $H^{1}(\Omega) \equiv \bigoplus_{i \in F} H^{1}\left(U_{i}\right)$. On the other hand, given any $\mu \in$ $m(K)$ by Corollary $3.12, \mu=\sum_{i \in I} \mu_{i}, \mu_{i} \in M\left(K_{i}\right), \mu_{i}, \mu_{j}$ are mutually singular; which implies that $\mu_{i}$ is real for all $i$, i.e., $\mu_{i} \in m\left(K_{i}\right)$ for every $i$ and by our assumption above

$$
\mu_{i}=0 \text { for } i \notin F \text {. }
$$

Thus the natural mapping $m(K) \rightarrow \bigoplus_{i \in F} m\left(K_{i}\right)$ is an isomorphism.

Thus by our hypothesis,

$$
H^{1}(\Omega) \equiv m(K) \text {. }
$$

4. Harmonic 1-forms, real boundary measures.

Lemma 4.1. Let $\omega$ be a holomorphic 1-form defined on an 
annulus $D=\{z ; \delta<|z|<1\}$. Assume that $\exists$ a real measure $\mu$ on $|z|=1$ such that for any continuous function $h$ on $D, \int_{|z|=r} h \omega \rightarrow$ $\int h d \mu$ as $r \rightarrow 1-0$. Then $\iint \omega \Lambda * \omega<\infty$ and for any $\mathscr{C}^{1}$-function $h$ defined on $D$, vanishing in a neighborhood of $|z|=\delta$ and $\iint_{D} d h \Lambda * d h<\infty, \iint_{D} d h \Lambda \operatorname{Im} \omega=0$.

(For the definition of $* \omega, \operatorname{Im} \omega$ see Ahlfors-Sario [3] p. 271.)

Proof. Since $\omega$ is a holomorphic 1-form, there exists a holomorphic function $g(z)$ on $D$ such that $\omega=g(z) d z$.

Let $\widetilde{D}$ denote the annulus $\delta<|z|<1 / \delta$, the double of $D$. Define $\tilde{\omega}$ a holomorphic 1-form on $\widetilde{D}$ in the following way. Define $\tilde{\omega}=$ $g(z) d z$ for $|z|<1$ and for $|z|>1$,

$\tilde{\omega}=-\widetilde{g}\left(\frac{1}{\bar{z}}\right) \frac{d z}{z^{2}}$. We note that $\tilde{\omega}$ is not defined on $|z|=1$.

By hypothesis, we obtain that there exists a constant $C$ such that $\int_{|z|=r}|\omega|<C$ for $r$ such that $(1+\delta) / 2 \leqq r<1$.

$$
\text { i.e., } \int_{|z|=r}|g(z)||d z| \leqq C \text {. }
$$

Thus if $g$ is defined as $g(z)$ on $|z|<1$ and $-\bar{g}(1 / \bar{z}) 1 / z^{2}$ on $|z|>1$, $g$ belongs $L^{1,10 c}(\widetilde{D})$. We shall now prove that $\partial \widetilde{g} / \partial \bar{z}=0$ in the sense of distributions.

Let $h$ be any $C^{\infty}$-function with compact support in $\widetilde{D}$. Then

$$
\begin{array}{r}
\iint_{\widetilde{D}} \frac{\partial h}{\partial \bar{z}} \widetilde{g}(z) d \bar{z} \Lambda d z=\iint_{\tilde{D}} d h \Lambda \widetilde{g}(z) d z=\iint_{\widetilde{D}} d h \Lambda \tilde{\omega} \\
=\lim _{\varepsilon \rightarrow 0} \int_{|z|=1-\varepsilon} h \omega-\int_{|z|=1+\varepsilon}-h \widetilde{g}\left(\frac{1}{\bar{z}}\right) \frac{d z}{z^{2}}
\end{array}
$$

(by Stoke's formula applied to the annulii $\delta<|z|<1-\varepsilon, 1+\varepsilon<$ $|z|<1 / \delta)$

$$
\begin{aligned}
& =\int h d \mu+\lim _{\varepsilon \rightarrow 0} \int_{|z|=1+\varepsilon} h \bar{g}\left(\frac{1}{\bar{z}}\right) \frac{d z}{z^{2}} \\
& =\int h d \mu-\lim _{\varepsilon \rightarrow 0} \int_{|z|=1 / 1+\varepsilon} h\left(\frac{1}{\bar{z}}\right) \bar{g}(z) d \bar{z} \\
& =\int h d \mu-\int \overline{h d \mu}=0 \text { since } \mu \text { is real . }
\end{aligned}
$$

Therefore we obtain that $g$ can be defined suitably on $|z|=1$ so that $g$ is holomorphic in all of $\widetilde{D}$. Hence $\iint_{(1+\tilde{o} / 2)<|z|<(2 / 1+\tilde{o})} \omega \Lambda * \omega<$ $\infty$ and consequently, 


$$
\iint \omega \Lambda * \omega<\infty
$$

$(1+\delta) / 2<|z|<1$.

Also for any real $h, \mathscr{C}^{1}$ on $\bar{D}$ and vanishing in a neighborhood of $|z|=\delta$,

$$
\iint_{D} d h \Lambda \omega=\int_{|z|=1} h \omega=\int h d \mu
$$

and so

$$
\operatorname{Im} \iint_{D} d h \Lambda \omega=\iint_{D} d h \Lambda \operatorname{Im} \omega=\iint_{D} d h \Lambda \operatorname{Im} \omega=\operatorname{Im} \int h d \mu=0 .
$$

Now given any $h, \mathscr{C}^{1}$ on $D$ and vanishing in a neighborhood of $|z|=\delta$, define $h_{\varepsilon}(z)=h(z /(1+\varepsilon))$. Then $h_{\varepsilon}$ is $\mathscr{C}^{1}$ on $\bar{D}$ for every $\varepsilon>0$ and vanishes in a neighborhood of $|z|=\delta$ and furthermore $\iint d h_{\varepsilon} \Lambda *$ $d h_{\varepsilon}<\infty$ and $\iint d\left(h-h_{\varepsilon}\right) \Lambda *\left(d h-d h_{\varepsilon}\right) \rightarrow 0$ as $\varepsilon \rightarrow 0$.

Hence, since we already know that $\iint_{D} d h_{\varepsilon} \Lambda \operatorname{Im} \omega=0$ for all $\varepsilon$ and $\iint \operatorname{Im} \omega \Lambda * \operatorname{Im} \omega<\infty$, we can take the limit under the integral sign and obtain that

$$
\iint_{D} d h \Lambda \operatorname{Im} \omega=0
$$

Lemma 4.2. Let $\omega$ be a holomorphic 1-form on $D=\{z ; \delta<$ $|z|<1\}$ such that $\iint_{D} \omega \Lambda * \omega<\infty$. Further assume that for any $h$, $\mathscr{C}^{1}$ on $D$ and vanishing in a neighborhood of $|z|=\delta$ and $\iint_{D} d h \Lambda *$ $d h<\infty, \iint_{D} d h \Lambda \operatorname{Im} \omega=0$.

Then $\exists$ a real measure $\mu$ on $|z|=1$ such that for any continuous function $h$ on $\bar{D}, \int_{|z|=r} h \omega \rightarrow \int h d \mu$ as $r \rightarrow 1-0$.

Proof. Let $\omega=g(z) d z$ for $\delta<|z|<1$ and $\tilde{\omega}$ be defined as $\omega$ on $\delta<|z|<1$ and

$$
=-\bar{g}\left(\frac{1}{\bar{z}}\right) \frac{d z}{z^{2}} \text { on } 1<|z|<\frac{1}{\delta} .
$$

By hypothesis, $\iint_{\delta<|z|<1 / \delta} \omega \Lambda * \omega<\infty$. We shall now establish that $\bar{\partial} \tilde{\omega}=0$ in the sense of distributions.

Let $h$ be any $\mathscr{C}^{1}$-function with compact support in $\delta<|z|<$ $1 / \delta$. Then 


$$
\begin{aligned}
& \iint_{\tilde{o}<|z|<1 / \bar{\sigma}} \bar{\partial} h \Lambda \tilde{\omega}=\iint d h \Lambda \tilde{\omega}=\lim _{r \rightarrow 1-0} \iint_{\tilde{o}<|z|<r} d h \Lambda \tilde{\omega}+\iint_{1 / r<|z|<1 / \delta} d h \Lambda \tilde{\omega} \\
& \quad=(\text { By Stoke's }) \lim _{r \rightarrow 1-0}\left(\int_{|z|=r} h \omega-\int_{|z|=1 / r} h \omega\right) \\
& \quad=\lim _{r \rightarrow 1-0}\left(\int_{|z|=r} h \omega-\int_{|z|=1 / r} h(z)\left(-\bar{g}\left(\frac{1}{\bar{z}}\right)\right) \frac{d z}{z^{2}}\right) \\
& \quad=\lim _{r \rightarrow 1-0}\left(\int_{|z|=r} h \omega-\int_{|z|=r} h\left(\frac{1}{\bar{z}}\right) \bar{g}(z) d z\right) \\
& \quad=\lim _{r \rightarrow 1-0} \int_{|z|=r} h \omega-\int_{|z|=r} h\left(\frac{1}{\bar{z}}\right) \bar{\omega} \\
& \quad=\lim _{r \rightarrow 1-0}\left(\int_{|z|=r}\left(h(z)-h\left(\frac{1}{\bar{z}}\right)\right) \operatorname{Re} \omega+i \int_{|z|=r}\left(\left(h(z)+h\left(\frac{1}{\bar{z}}\right) \operatorname{Im} \omega\right)\right) .\right.
\end{aligned}
$$

Since $h(z)+h(1 / \bar{z})$ vanishes in a neighborhood of $|z|=\delta$ and $\iint_{D} d h \Lambda * d h<\infty$, we have, by hypothesis,

$$
\iint d h \Lambda \operatorname{Im} \omega=0=\iint d h\left(\frac{1}{\bar{z}}\right) \Lambda \operatorname{Im} \omega \text { i.e., }
$$

(By Stoke's)

$$
\lim _{r \rightarrow 1-0} \iint_{\tilde{\delta}<|z|<r} d h \Lambda \operatorname{Im} \omega=\lim _{r \rightarrow 1-0} \int_{|z|=r} h \operatorname{Im} \omega=0 .
$$

Hence

$$
\begin{aligned}
& \iint_{\tilde{o}<|z|<1 / \delta} \bar{\partial} h \Lambda \tilde{\omega}=\lim _{r \rightarrow 1-0} \int_{|z|=r}\left(h(z)-h\left(\frac{1}{\bar{z}}\right)\right) \operatorname{Re} \omega \\
& \quad=\lim _{r \rightarrow 1-0} \iint_{\tilde{\delta}<|z|<r} d\left(h(z)-h\left(\frac{1}{z}\right)\right) \Lambda \operatorname{Re} \omega \text { (By Stoke's). }
\end{aligned}
$$

Since $h(z)-h(1 / \bar{z}) \in H_{2}(D)$ (here it denotes the Sobolev space) and vanishes on $\partial D$, we find that

$h(z)-h(1 / \bar{z}) \in \stackrel{\circ}{H}_{2}(D)$ (see Agmon [1], p. 131, Lemma 9.10). But $\iint_{D} d h \Lambda \operatorname{Re} \omega=0$ for any $h$ that is $\mathscr{C}^{1}$ and has compact support in $D$ and hence for any $h$ in $\stackrel{H}{2}_{2}^{1}(D)$.

Therefore $\bar{\partial} \tilde{\omega}=0$. Hence $\tilde{\omega}$ is a holomorphic 1-form on $\delta<|z|<$ $1 / \delta$ which implies that $\int_{|z|=r}|g(z)||d z|$ is bounded as $r \rightarrow 1-0$. That means that $\omega$ defines a real boundary measure on $|z|=1$.

THEOREM 4.3. Borrowing the notation of Corollary $3.12, m\left(K_{i}\right) \equiv$ $H^{1}\left(U_{i}\right)$ for every $i$.

Proof. Let $\Gamma\left(U_{i}\right)$ denote the set of all holomorphic 1-forms $\omega$ 
such that $\iint_{U_{i}} \omega \Lambda * \omega<\infty$ and for any $\mathscr{C}^{1}$-function $h$ on $U_{i}$ such that $\iint d h \Lambda * d h<\infty, \iint e h \Lambda \operatorname{Im} \omega=0$.

The fact that $H^{1}\left(U_{i}\right) \equiv \Gamma\left(U_{i}\right)$ is well-known and can be found in Ahlfors-Sario [2], p. 284-288. Thus we need only prove that $m\left(K_{i}\right) \equiv \Gamma\left(U_{i}\right)$.

Let $\Delta_{i j}(1 \leqq j \leqq N(i))$ be the annulii as introduced in Theorem 3.6. Now if $\omega$ is a holomorphic 1-form on $U_{i}$ whose boundary measure is real, then $\omega \mid \Delta_{i j} \in \mathscr{H}\left(\Delta_{i j}\right)$ and further its boundary measure $\mu_{i j}$ on $\partial U_{i} \cap \partial \Delta_{i j}$ is real. We can apply now Lemma 4.1 to $\omega \mid \Delta_{i j}$ and obtain $\iint_{\Delta_{i i}} \omega \Lambda * \omega<\infty$ and $\iint_{\Delta_{i j}} d h \Lambda \operatorname{Im} \omega=0$ provided $h$ is a $\mathscr{C}^{1}$-function vanishing in a neighborhood of $\partial \Delta_{i j}-\partial U_{i}$. Thus using partition of unity, we obtain that $\iint_{U_{i}} \omega \Lambda * \omega<\infty$ and $\iint_{U_{i}} d h \Lambda \operatorname{Im} \omega=$ 0 for any $h, \mathscr{C}^{1}$ on $U_{i}$ and $\iint d h \Lambda * d h{ }^{i}<\infty$.

Now assume that $\omega \in \Gamma\left(U_{i}\right)$. Now $\omega \mid \Delta_{i j}$ satisfies the following conditions: $\iint_{\Lambda_{i j}} \omega \Lambda * \omega<\infty$ and any $\mathscr{C}^{1}$-function $h$ vanishing in a neighborhood of $\partial \Delta_{i j}-\partial U_{i}$ and $\iint_{\Delta_{i j}} d h \Lambda * d h<\infty, \iint d h \Lambda \operatorname{Im} \omega=0$. This is easily obtained by defining $h \stackrel{\Delta_{i j}}{=} 0$ on $U_{i}-\Delta_{i j}$. Now we can apply Lemma 4.2 to obtain that the boundary measure $\mu_{i j}$ of $\omega$ on $\partial \Delta_{i j} \cap \partial U_{i}$ is real. Since boundary measure $\mu_{i}$ of $\omega$ is $\sum_{j=1}^{N(i)} \mu_{i j}$ by Theorem 3.6, $\mu_{i}$ is real.

THEOREM 4.4. $m(K) \equiv H^{1}(\Omega)$.

Proof. It is immediate from Corollary 3.13 and Theorem 4.3.

5. A natural basis for $\mathscr{C}(\partial K) / \overline{\operatorname{Re} \mathscr{R}(K)}$ (Theorem 1.2). We may assume without loss of generality that $X$ is a noncompact surface with analytic boundary and $K$ a compact subset of $X$ such that $X-K$ has only finitely many connected components none of which is relatively compact. By Theorem 2.1, the canonical homomorphism $H_{1}(\stackrel{\circ}{K}) \rightarrow H_{1}(X)$ is injective.

Let $\gamma_{i}(1 \leqq i \leqq k)$ be a homology basis for $\stackrel{\circ}{K}$ and $\gamma_{i}(1 \leqq i \leqq k+l)$ be a homology basis for $X$. Let $\Theta$ denote the space of all harmonic functions $h$ on $X$ such that $\iint d h \Lambda * d h<\infty$.

We contend that given any $\sum a_{i} \gamma_{i} \neq 0, a_{i}$ real, there exists $h \in \Theta$ such that

$$
\int_{\Sigma a_{\imath} \gamma_{i}} * d h \neq 0 . \quad \text { Assume the contrary . }
$$

Then there exists a harmonic differential $\sigma$ with compact support (see Ahlfors-Sario [3], p. 288) such that 


$$
\int_{\sum a_{i^{\gamma} i}} * d h=\iint \sigma \Lambda * d h
$$

and so

$$
\iint \sigma \Lambda * d h=0 \forall h \in \Theta
$$

i.e. $* \sigma$ also has compact support. But $\sigma-i * \sigma$ is a holomorphic 1-form and it can not have compact support unless $\sigma=i * \sigma \equiv 0$ which implies $\sum a_{i} \gamma_{i}$ is homologous to zero.

This proves that the mapping $\psi: \ominus \rightarrow R^{k+l}$ given by $\psi(h)=$ $\left(\int_{r_{1}} * d h, \cdots, \int_{r_{k+l}} * d h\right)$ is a surjection. Now let us pick $h_{i} \in \Theta$ such that $\int_{\gamma_{i}} * d h_{i}=1$ and $\int_{\gamma_{j}} * d h_{i}=0$ for $j \neq i$.

We claim now that $h_{1}, h_{2} \cdot, h_{k}$ form a basis of $\mathscr{C}(\partial K)$ modulo $\overline{\operatorname{Re} \mathscr{R}(K)}$. Assume $\sum a_{i} h_{i} \in \overline{\operatorname{Re} \mathscr{R}(K)}$. Then there exists a function $f$ holomorphic in a neighborhood of $K$ such that $\left|\sum a_{i} h_{i}-\operatorname{Re} f\right|<\varepsilon$ on $\partial K$.

Since $\gamma_{i}$ lie in $\dot{K}$ for $1 \leqq i \leqq k$, and $\int_{\gamma_{j}}\left|\sum a_{i} * d k_{i}-\operatorname{Im} d f\right|<C \varepsilon$ where $C$ depends only on $\gamma_{j}$.

Since $\int_{\gamma_{j}} d f=0$ and $\int_{r_{j}} * d h_{i}=\delta_{i j}$ (Kronecker $\delta$ ), we obtain that $\left|a_{i}\right|<C \varepsilon$ for $1 \leqq i \leqq k$. Since this is true for all $\varepsilon>0, a_{i}=0 \forall i$. Thus $\left\{h_{i}\right\}_{1 \leqq i \leqq k}$ are linearly independent modulo $\overline{\operatorname{Re} \mathscr{R}(K)}$ and because $\operatorname{dim} \mathscr{C}(\partial K) \overline{\operatorname{Re} \mathscr{R}(K)}=k$, we have that $\left\{h_{i}\right\}_{1 \leqq i \leqq k}$ is a basis for $\mathscr{C}(\partial K) /$ $\overline{\operatorname{Re} \mathscr{R}(K)}$.

Note: Theorems 1.1 and 1.2 for plane domains are published by us in the Journal of Approximation Theory, Vol. 30, No. 1, 1980 under the title "The Rational Defect of a Plane Domain."

\section{REFERENCES}

1. S. Agmon, Lectures on Elliptic Boundary Value Problems, Van Nostrand Company, Princeton, 1963.

2. P. R. Ahern and D. Sarason, On some hypo-dirichlet algebras of analytic functions. Amer. J. Math, 89 (1967), 932-941.

3. Lars. V. Ahlfors and Leo Sario, Riemann Surfaces, Princeton Univ. Press, Princeton, 1960 .

4. Erret Bishop, Boundary measures of analytic differentials, Duke Math. J., 27 (1960), 331-340.

5. I. Glicksberg, Dominant representing measures and rational approximation, TAMS, 130 (1968), 425-462.

6. G. M. Goluzin, Geometric theory of functions of one complex variable, Nauka, Moscow, 1966.

7. Laura Ketchum Kodama, Boundary measures of analytic differntixls and uniform approximation on a Riemann surface, Pacific J. Math., 15 (1965), 1261-1277. 
8. R. Narasimhan, Imbedding of Riemann surfaces, Gottingen Nachrichten No. 7 (1960), 159-165.

9. J. L. Walsh, The approximation of harmonic functions by harmonic polynomials and harmonic rational functions, Bull. AMS, 35 (1929), 499-544.

10, A. Zygmund, Trigonometric Series, vol. I, Cambridge University Press, Cambridge, 1959.

Received April 16, 1979.

UNIVERSITY OF WISCONSIN

MADISON, WI 53706

AND

The UNIVERSITY OF TOLEDO

TOLEDO, OH 43606 


\section{PACIFIC JOURNAL OF MATHEMATICS}

EDITORS

DONALD BABBITT (Managing Editor)

University of California

Los Angeles, CA 90024

Hugo RossI

University of Utah

Salt Lake City, UT 84112

C. C. MOORE and ANDREW OGG

University of California

Berkeley, CA 94720

\section{J. DugundjI}

Department of Mathematics

University of Southern California

Los Angeles, CA 90007

R. FINN and J. MILGRAM

Stanford University

Stanford, CA 94305

\section{ASSOCIATE EDITORS}
R. ARENS
E. F. BECKENBACH
B. H. NEUManN
F. WOLF
K. YoSHIDA

\section{SUPPORTING INSTITUTIONS}

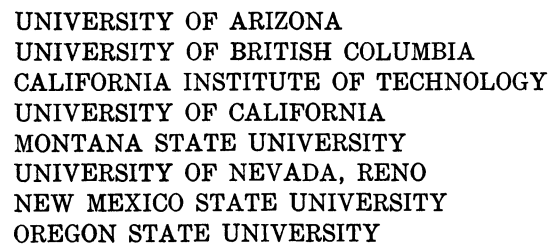

UNIVERSITY OF ARIZONA

UNIVERSITY OF BRITISH COLUMBIA

CALIFORNIA INSTITUTE OF TECHNOLOGY

UNIVERSITY OF CALIFORNIA

MONTANA STATE UNIVERSITY

UNIVERSITY OF NEVADA, RENO

NEW MEXICO STATE UNIVERSITY

OREGON STATE UNIVERSITY

\author{
UNIVERSITY OF OREGON \\ UNIVERSITY OF SOUTHERN CALIFORNIA \\ STANFORD UNIVERSITY \\ UNIVERSITY OF HAWAII \\ UNIVERSITY OF TOKYO \\ UNIVERSITY OF UTAH \\ WASHINGTON STATE UNIVERSITY \\ UNIVERSITY OF WASHINGTON
}

The Supporting Institutions listed above contribute to the cost of publication of this Journal, but they are not owners or publishers and have no responsibility for its content or policies.

Mathematical papers intended for publication in the Pacific Journal of Mathematics should be in typed form or offset-reproduced, (not dittoed), double spaced with large margins. Please do not use built up fractions in the text of the manuscript. However, you may use them in the displayed equations. Underline Greek letters in red, German in green, and script in blue. The first paragraph or two must be capable of being used separately as a synopsis of the entire paper. Please propose a heading for the odd numbered pages of less than 35 characters. Manuscripts, in triplicate, may be sent to any one of the editors. Please classify according to the scheme of Math. Reviews, Index to Vol. 39. Supply name and address of author to whom proofs should be sent. All other communications should be addressed to the managing editor, or Elaine Barth, University of California, Los Angeles, California, 90024.

50 reprints to each author are provided free for each article, only if page charges have been substantially paid. Additional copies may be obtained at cost in multiples of 50 .

The Pacific Journal of Mathematics is issued monthly as of January 1966. Regular subscription rate: $\$ 102.00$ a year (6 Vols., 12 issues). Special rate: $\$ 51.00$ a year to individual members of supporting institutions.

Subscriptions, orders for numbers issued in the last three calendar years, and changes of address shoud be sent to Pacific Journal of Mathematics, P.O. Box 969, Carmel Valley, CA 93924, U.S.A. Old back numbers obtainable from Kraus Periodicals Co., Route 100, Millwood, NY 10546.

\section{PUBLISHED BY PACIFIC JOURNAL OF MATHEMATICS, A NON-PROFIT CORPORATION}

Printed at Kokusai Bunken Insatsusha (International Academic Printing Co., Ltd.). 8-8, 3-chome, Takadanobaba, Shinjuku-ku, Tokyo 160, Japan. 


\section{Pacific Journal of Mathematics}

\section{Vol. 97, No. $2 \quad$ February, 1981}

Patrick Robert Ahern and N. V. Rao, A note on real orthogonal measures . . . . . 249

Kouhei Asano and Katsuyuki Yoshikawa, On polynomial invariants of fibered

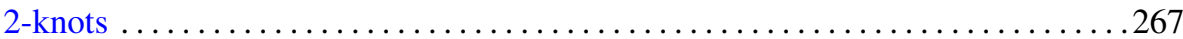

Charles A. Asmuth and Joe Repka, Tensor products for $S L_{2}(\mathscr{K})$. I.

Complementary series and the special representation

Gary Francis Birkenmeier, Baer rings and quasicontinuous rings have a

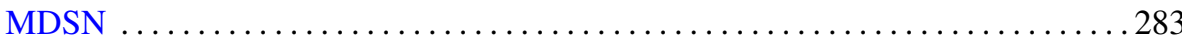

Hans-Heinrich Brungs and Günter Törner, Right chain rings and the generalized

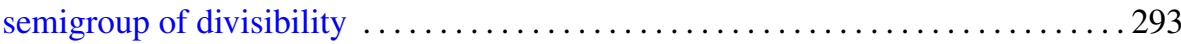

Jia-Arng Chao and Svante Janson, A note on $H^{1} q$-martingales . . . . . . . . . 307

Joseph Eugene Collison, An analogue of Kolmogorov's inequality for a class of

additive arithmetic functions

Frank Rimi DeMeyer, An action of the automorphism group of a commutative ring on its Brauer group

H. P. Dikshit and Anil Kumar, Determination of bounds similar to the Lebesgue

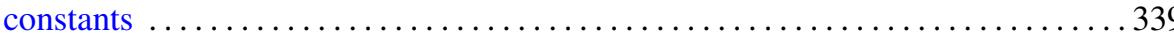

Eric Karel van Douwen, The number of subcontinua of the remainder of the plane

D. W. Dubois, Second note on Artin's solution of Hilbert's 17th problem. Order spaces

Daniel Evans Flath, A comparison of the automorphic representations of GL(3) and its twisted forms

Frederick Michael Goodman, Translation invariant closed $*$ derivations

Richard Grassl, Polynomials in denumerable indeterminates

K. F. Lai, Orders of finite algebraic groups

George Kempf, Torsion divisors on algebraic curves

Arun Kumar and D. P. Sahu, Absolute convergence fields of some triangular matrix methods

Elias Saab, On measurable projections in Banach spaces

Chao-Liang Shen, Automorphisms of dimension groups and the construction of AF algebras

Barry Simon, Pointwise domination of matrices and comparison of $\Phi_{p}$ norms

Chi-Lin Yen, A minimax inequality and its applications to variational inequalities

Stephen D. Cohen, Corrections to: "The Galois group of a polynomial with two indeterminate coefficients"

Phillip Schultz, Correction to: "The typeset and cotypeset of a rank 2 abelian group"

Pavel G. Todorov, Correction to: "New explicit formulas for the $n$th derivative of composite functions"

Douglas S. Bridges, Correction to: "On the isolation of zeroes of an analytic function" 